\title{
Perceived size of familiar objects and the theory of off-sized perceptions
}

\author{
JOHN PREDEBON \\ University of Sydney, Sydney, Australia
}

\begin{abstract}
In three experiments, I examined the claim (Gogel, 1969; Gogel \& Newton, 1969) that familiar objects viewed under reduced stimulus conditions frequently appear to be off-sized (i.e., larger or smaller than normal). In Experiments 1 and 2, I presented images of different familiar objects, one at a time, at distances of 1 and $2 \mathrm{~m}$ from the observers. The images were normal-, large-, or small-sized versions of familiar objects, and the observers judged the perceived size of each object relative to its familiar normal size. In Experiment 3, I presented normal-, large-, and smallsized versions of the same familiar object at physical distances of 1 and $2 \mathrm{~m}$. The pattern of size results was similar across the three experiments. In general, normal-sized objects appeared normalto small-sized at the 1-m distance and small-sized at the 2-m distance; small-sized objects appeared small-sized at the 1-m distance and even smaller at the 2-m distance; and large-sized objects appeared normal- to large-sized at the 1-m distance and normal- to small-sized at the 2-m distance. The distance results of Experiment 3 indicated that familiar size was an effective determinant of reported distance. These results are consistent with Gogel's theory of off-sized perceptions and, more generally, with the claim that familiar size is not an important determinant of perceived size.
\end{abstract}

It is now well established that under conditions of reduced visual stimulation, the familiar size of an object influences direct reports (e.g., verbal estimates) of its size and distance (for review, see Sedgwick, 1986). There are two different approaches to explaining this finding. Perhaps the most widely accepted explanation-hereafter referred to as the familiar-size theory-claims that familiar size influences perceived size, which then, in agreement with the size-distance invariance hypothesis (SDIH), determines perceived distance (Ittelson, 1960). According to this theory, a physically off-sized familiar object will appear to be normal-sized and, consequently, perceptually localized at the distance at which its normal-sized version would have to be positioned in order to match the angular size of the off-sized object.

The other explanation-referred to as the theory of offsized perceptions (Gogel, 1976; Gogel \& Da Silva, 1987) - asserts that familiar size is not a major determinant of either perceived size or perceived distance. Rather, the perceived distance of a familiar object viewed under visually impoverished conditions is determined by the egocentric reference distance (ERD), which in turn determines the object's perceived size in the manner specified by the SDIH. The particular value of the ERD's prevailing in any given situation is dependent on the individual observer's specific distance tendency (the tendency to perceive an object to be at a distance of about 2-3 m, re-

This research was funded by an Australian Research Council grant. The data of Experiments 1 and 3 were collected by Jacob S. Woolley. Address correspondence to J. Predebon, University of Sydney, Department of Psychology, Sydney, NSW, 2006, Australia (e-mail: john@ psychvax.psych.su.oz.au). gardless of its actual distance) and on the unavoidably present residual oculomotor cues to distance (see Gogel \& Da Silva, 1987).

It follows from Gogel's theory of off-sized perceptions that the perceived size of a familiar object need not correspond to its characteristic normal size, in which case an off-sized judgment or an off-sized perception occurs. A physically normal-sized object, for example, will appear normal-sized only if its perceived distance (ERD) corresponds to its physical distance; if it is presented closer or farther than the ERD, the object will appear larger and smaller than its normal size, respectively. The theory explains the effect of familiar size on direct reports of distance by assuming that off-sized perceptions allow observers to infer, or make a cognitive response to, the object's distance. Specifically, given the perspective bias (Carlson, 1977) - that is, the widespread belief that the apparent size of an object of constant physical size varies inversely with its distance-observers are likely to assume that a familiar object that appears smaller (larger) than normal is a physically normal-sized object at a greater (shorter) distance than the distance at which it is perceived to be (Gogel \& Da Silva, 1987).

Both theories are consistent with findings indicating that familiar size influences direct reports of distance. These findings, therefore, cannot discriminate between the two theories unless independent evidence is available to resolve the reports into cognitive and perceptual components. However, the two theories make different predictions concerning the occurrence of off-sized perceptions: the theory of off-sized perceptions, but not the familiarsize theory, necessarily predicts that, under reduced-cue conditions, familiar objects frequently appear off-sized. 
The most persuasive evidence for the notion of off-sized perceptions comes from the work of Gogel (1969) and Gogel and Newton (1969). The stimuli in these studies were images depicting off-sized versions of 16 different familiar objects; the width of the image compared with the object's normal width varied from .05 to 2.42 . The stimuli were presented one at a time at a distance of $133 \mathrm{~cm}$, and observers reported the perceived size of each familiar object relative to its remembered normal size. Size judgments were strongly influenced by the actual size of the image and only weakly by the objects' familiar size-that is, the objects often appeared to be off-sized. These results do not provide convincing support for the theory of off-sized perceptions, however. Gogel and Newton also found that distance estimates $(165 \mathrm{~cm})$ of an unfamiliar object were similar to its physical distance of presentation $(133 \mathrm{~cm})$, so the possibility that residual distance cues were effective in counteracting or minimizing the expected effect of familiar size on perceived size cannot be discounted. ${ }^{1}$ Clearly, the important issue of whether or not familiar objects are perceived to be off-sized under conditions that exclude or control for the possible contribution of residual distance cues remains unresolved.

The main aim of the three experiments presented here was to evaluate the predictive utility of the theory of offsized perceptions and the familiar-size theory with respect to the effect of familiar size on perceived size. The experiments were similar to Gogel and Newton's (1969) study, except for two important respects-all of their stimuli were off-sized versions of familiar objects, but in the present investigation 1 also included normal-sized objects, presented at two distances ( 1 and $2 \mathrm{~m}$ ) rather than one. Of primary interest was the effect of changing physical distance or, equivalently, visual angle on the frequency of reports of off-sized perceptions for each of the three different size categories (large, small, normal) of familiar objects.

The theory of off-sized perceptions predicts a systematic effect of visual angle on the pattern of size responses to the three different size categories of objects. Under reduced-cue conditions, objects are localized at the ERD irrespective of their physical distance (1 or $2 \mathrm{~m}$ ) of presentation. It follows from the SDIH that the objects' perceived sizes will be related to their visual angle sizes. In particular, given the inverse relationship between visual angle and physical distance, the same object must appear smaller when presented at the 2-m distance than at the $1-\mathrm{m}$ distance. This relationship between the perceived size of an object and its physical distance (i.e., visual angle) has predictable consequences on the pattern of off-size responses to the physically small-, normal-, and largesized objects. As an example, suppose the ERD is $2 \mathrm{~m}$. In this case, the size and distance of a normal-sized object at the $2-\mathrm{m}$ distance will be perceived correctly and, hence, reported as normal-sized. If the same object is presented at the 1-m distance, it will be perceived at $2 \mathrm{~m}$ (ERD) and, therefore, will appear and be reported as larger than normal-sized. Similarly, a large-sized object will be perceived veridically as being larger than normal at the 2-m distance and even larger at the $1-\mathrm{m}$ distance, and a small-sized object will be perceived correctly as being smaller than normal at the 2-m distance and less small or even normal- to large-sized when presented at the 1-m distance. In general, while the particular pattern of offsized judgments will depend on the ERD's prevailing under the conditions of the present experiments, the theory predicts a systematic dependency of reports of offsized perceptions on the physical distance (visual angle) and on the category size of the objects. ${ }^{2}$

The familiar-size theory predicts little or no effect of visual angle on the pattern of size responses. Two versions of this theory need to be considered, however. The "strong" version assumes that familiar size is fully effective, and, consequently, that the objects will appear normal-sized regardless of whether they are physically normal- or off-sized and regardless of their physical distance of presentation. The "weak" version assumes that the unavoidably present residual distance cues (e.g., accommodation and convergence) are likely to attenuate the influence of familiar size. Hence, a potential conflict exists between the residual distance cues, on the one hand, and the familiar-size cue, on the other, in determining the perceived size of the off-sized objects. This version predicts that familiar size will be somewhat effective at both physical distances; the perceived size of the off-sized objects will "regress" or approach the objects' normal sizes with the extent of the regression directly related to the effectiveness of familiar size. For the normal-sized objects, a cue conflict does not exist, and, therefore, they should appear to be normal-sized at both distances of presentation.

Although it was not feasible in the present investigation to measure the possible perceived size "regression" of the off-sized objects, the weak form of the theory would be supported if the off-sized and normal-sized objects were reported to be off-sized and normal-sized, respectively. However, such an outcome could equally be attributed to the effectiveness of residual distance cues in determining the actual size of the objects. In Experiment 1, therefore, independent evidence for the effectiveness of the residual distance cues present in the display was also obtained. One group of observers judged the size and distance of an unfamiliar object at the 1-m distance, and another independent group judged the size and distance of an equivalent, angular-sized unfamiliar object at the 2-m distance. If residual distance cues are effective, there should be a significant difference between the size and/or distance estimates of the two objects.

The stimuli of Experiments 1 and 2 were different familiar objects, each of which was either physically normalor off-sized. Certain aspects of the findings of these experiments prompted Experiment 3, in which differentsized versions (small, normal, large) of the same familiar object were used. Additionally, only size judgments were obtained in Experiments 1 and 2, whereas distance judgments were included in Experiment 3. 


\section{EXPERIMENT 1}

In Experiment 1, observers reported on whether each successively presented familiar object appeared to be normal- or off-sized and, if the latter, estimated the perceived size of the object as a proportion of its remembered normal size. Distance judgments were not obtained for two reasons-to duplicate Gogel and Newton's (1969) procedure, and to minimize cognitive biases influencing the size reports. With respect to the latter, directing attention to both the size and distance of the objects is likely to elicit the perspective bias with the consequence that observers may attribute the discrepancy between the perceived and the remembered size of the familiar object to its (inferred) distance. In this event, a cognitive response to size is likely to occur; observers will assume, and report, the object to be normal-sized even though it is perceived to be off-sized (Carlson, 1977; Gogel, 1969). Since the primary aim of the present experiment was to assess the dependency of perceived size on visual angle and, more generally, to determine the frequency of off-sized perceptions, it seemed prudent to minimize the occurrence of cognitive responses to size that otherwise might occur if distance judgments were obtained.

\section{Method}

Observers. The observers were 50 undergraduate volunteers, all of whom were given course credit for their participation in the experiment. All the observers had normal or corrected vision, and all were unaware of the purpose of the experiment.

Apparatus and Stimuli. The stimuli were photographic color transparencies of nine different familiar objects. Three objects were normal-sized, three were smaller, and three were larger than normal. Table 1 lists the familiar objects used in the experiment, the normal widths of the objects, the widths of the images of the objects on the transparency, the ratio of the width of the image to the normal width of the object, and the visual angle of the image at the 1-m distance. Each transparency was attached to a milk glass screen of a light-tight box, and was transilluminated by a fluorescent light source in the box. The stimuli were positioned either $1 \mathrm{~m}$ or $2 \mathrm{~m}$ (the maximum distance possible in the visual chamber) from the observer. There also were two square, blank transparencies:

Table 1

Characteristics of Stimuli Used in Experiments 1 and 2

\begin{tabular}{lrrrr}
\hline \multicolumn{1}{c}{ Object } & $\begin{array}{c}\text { Width of } \\
\text { Image* }\end{array}$ & $\begin{array}{c}\text { Normal } \\
\text { Width* }\end{array}$ & $\begin{array}{c}\text { Ratio } \\
\text { (Image Width to } \\
\text { Normal Width) }\end{array}$ & $\begin{array}{c}\text { Visual Angle } \\
\text { (in Degrees) } \\
\text { at 1 m }\end{array}$ \\
\hline \multicolumn{5}{c}{ Small-Sized } \\
Tennis ball & 4.6 & 6.5 & .71 & 2.63 \\
Ruler & 15.0 & 30.0 & .50 & 8.53 \\
\$5 bill & 8.7 & 15.0 & .58 & 4.97 \\
& \multicolumn{5}{c}{ Normal-Sized } \\
Bankcard & 8.5 & 8.5 & 1.00 & \\
Playing card & 5.7 & 5.7 & 1.00 & 3.86 \\
Envelope & 14.5 & 14.5 & 1.00 & 8.26 \\
& \multicolumn{5}{c}{ Large-Sized } \\
Golf ball & 5.7 & 4.2 & 1.36 & \\
Key & 11.1 & 5.4 & 2.05 & 3.26 \\
Stamp & 5.3 & 2.7 & 1.96 & 3.33 \\
\hline
\end{tabular}

*In centimeters. one was $5 \times 5 \mathrm{~cm}$ and the other was $10 \times 10 \mathrm{~cm}$. The small and large blanks were viewed from a distance of 1 and $2 \mathrm{~m}$, respectively, and thus had identical angular sizes. The stimuli were presented in a light-tight visual chamber, and were centered with respect to the observer's right eye. Baffle screens and black cloth material were used to eliminate stray light and to ensure that the observers only saw the stimulus against a completely darkened surround. The average luminance of the transparencies was $.27 \mathrm{~cd} / \mathrm{m} .{ }^{2}$ At the observation end of the chamber there was an upright panel with an aperture containing a cylindrical reduction tube.

Design and Procedure. There were two groups of 25 observers each. One group monocularly viewed the stimuli from a distance of $1 \mathrm{~m}$, and the other group from a distance of $2 \mathrm{~m}$.

All the observers were first presented with one of the two blank stimuli: 12 and 13 of the observers, assigned to the 1-m and 2-m distance conditions, respectively, were presented with the 5-cm blank at the $1-\mathrm{m}$ distance, while the remaining 25 observers were presented with the $10-\mathrm{cm}$ blank at the $2-\mathrm{m}$ distance. The observer's head was positioned in a head- and chinrest device with the right eye in front of the closed viewing aperture. Then the room lights were switched off and, after a 15-sec period, the aperture was opened to reveal the blank stimulus. The observer's task was to indicate, in centimeters, meters, or a combination of both, the perceived width and the perceived distance of the blank. Then the aperture was closed and the observer left the room, during which time the experimenter arranged the stimulus display for the next trials.

The observers were instructed that they would be making size judgments of a series of familiar objects. The instructions were similar to those used by Gogel and Newton (1969): "Many of the familiar objects we encounter have a characteristic size or restricted range of sizes. ... It is possible, however, to make unusual sized objects. ... Such unusual sizes of familiar objects are referred to as off-sized objects to distinguish them from the same familiar object of more normal size. Your task in this experiment is to tell me whether the objects I am going to show you appear normalsized or off-sized. . . A All, some or none of the objects may appear off-sized. ... Try to make your responses as rapidly as you can using as your criterion how large or how small the object appears to you relative to the normal size of that particular object." The observers were told that if the object appeared to be off-sized, they were to give a number indicating how much bigger or how much smaller the object appeared to be relative to a normal-sized object of the same kind, with the number 100 assigned to the latter. After the observer had indicated an understanding of the magnitude estimation task, he/she was seated in the observation end of the visual chamber and his/her head was positioned in the headand chinrest device. Then the room lights were switched off and, after a 15 -sec period, the aperture was opened. The observer was asked to identify the object, to indicate whether it appeared normalor off-sized, and, if it appeared off-sized, to give a magnitude estimate of how big or small the object appeared to be relative to its normal or standard size. The aperture was then closed and the observer left the room while the experimenter replaced the stimulus, after which the observer returned and the procedure was repeated. There were 10 trials in all, 1 blank stimulus trial followed by the 9 familiar-object trials, with an intertrial interval of approximately $3 \mathrm{~min}$. The order of presentation of the familiar objects was randomized across observers, with the restriction that each object be presented first an approximately equal number of times.

\section{Results}

Each observer's magnitude estimate of the size of each familiar object was divided by 100 -the value assigned to the normal size of the object-to give a ratio of perceived size relative to the object's normal size. Table 2 shows the means, standard deviations, and medians of the 
Table 2

Means, Standard Deviations, and Medians of Ratios of the Perceived Size of Each Familiar Object Relative to its Normal Size for the 25 Observers in Each of the Two Distance Conditions of Experiment 1

\begin{tabular}{|c|c|c|c|c|c|c|}
\hline & \multicolumn{6}{|c|}{ Distance of Object } \\
\hline & \multicolumn{3}{|c|}{$1 \mathrm{~m}$} & \multicolumn{3}{|c|}{$2 \mathrm{~m}$} \\
\hline & $M$ & $S D$ & Median & $M$ & $S D$ & Median \\
\hline \multicolumn{7}{|c|}{ Small-Sized } \\
\hline Tennis ball & .55 & .28 & .50 & .46 & .19 & .40 \\
\hline Ruler & .51 & .23 & .50 & .37 & .22 & .35 \\
\hline$\$ 5$ bill & .54 & .27 & .50 & .43 & .26 & .40 \\
\hline \multicolumn{7}{|c|}{ Normal-Sized } \\
\hline Bankcard & .91 & .29 & 1.00 & .75 & .26 & .75 \\
\hline Playing card & .87 & .21 & .95 & .72 & .26 & .75 \\
\hline Envelope & .86 & .32 & .78 & .63 & .25 & .63 \\
\hline \multicolumn{7}{|c|}{ Large-Sized } \\
\hline Golf ball & 1.06 & .24 & 1.00 & .89 & .20 & .98 \\
\hline Key & 1.17 & .30 & 1.00 & .98 & .27 & 1.00 \\
\hline Stamp & 1.27 & .44 & 1.18 & .95 & .29 & 1.00 \\
\hline
\end{tabular}

ratio scores; a ratio of 1 indicates that the object was perceived to be normal-sized, and ratios greater and less than 1 indicate that the object was perceived to be larger and smaller, respectively, than its normal size. Table 3 shows the frequency with which the 25 observers of each distance condition reported the objects to be normal- (ratio $=1$ ), large- (ratio $>1$ ), and small- (ratio $<1$ ) sized.

As is evident from the data in Table 2, the objects frequently were perceived to be off-sized. In general, the pattern of results shows a dependency of the size judgments on category size (large, normal, small) and on physical distance. On average, the normal-sized objects appeared normal-sized to slightly smaller than normal-sized at the 1-m distance and smaller at the 2-m distance; the small-sized objects appeared smaller than normal-sized at the 1-m distance and even smaller at the 2-m distance; and the large-sized objects appeared normal to slightly larger than normal-sized at the 1-m distance and normal to slightly smaller than normal at the $2-\mathrm{m}$ distance. These trends also are reflected in the frequency data of Table 3. For example, the normal-sized objects were perceived to be normal-sized more frequently at the 1-m distance, and the large-sized objects were perceived to be large-sized more frequently at the $1-\mathrm{m}$ distance.

The results of a 3 (stimuli) $\times 2$ (distance) mixed-design analysis of variance (ANOVA), performed separately on the ratio data of the normal-, small-, and large-size category conditions, were consistent with these observations. The main effect of distance was significant in the analysis of the normal- and large-object data, and marginally nonsignificant in the analysis of the small-object data $[F(1,48)=8.58,10.35, p<.05 ; 3.85, p=.06$, respectively]. The main effect of stimuli was significant only in the analysis of the large-object condition $[F(2,96)=$ $5.88, p<.05]$. None of the interactions was significant. Identical outcomes were obtained with the ANOVAs performed on the log transformation of the magnitude size estimates.
In the present investigation, residual oculomotor distance cues were potentially available to specify the actual size and distance of the objects. If residual distance cues were effective, then the majority of the size reports should mirror the actual sizes (large, normal, small) of the objects. This obviously did not occur. In any event, the results of the "blank" conditions suggest that residual distance cues were at best only weak determinants of perceived size and distance. If residual distance cues were effective, then the two identical angular-sized blanks should appear different in size and at different distances. The means and confidence limits (in parentheses) of the size and distance estimates of the 5-cm target at the $1-\mathrm{m}$ distance were $3.7( \pm .92) \mathrm{cm}$ and $59.2( \pm 21.43) \mathrm{cm}$, respectively, and the corresponding means of the $10-\mathrm{cm}$ target at the 2-m distance were $4.4( \pm 1.09) \mathrm{cm}$ and 72.4 $( \pm 20.98) \mathrm{cm}$. The difference between the two distance conditions was not significant for either the size estimates or the distance estimates $[t(48)=.94, .91, p>.05$, respectively], indicating that residual distance cues were not very effective determinants of the actual size and distance of the stimuli. It cannot be concluded, however, that residual distance cues were completely ineffective, since both the size and distance estimates of the 2-m target were larger than the corresponding estimates of the 1-m target. Even so, the distance estimates suggest that the 2-m stimulus was perceived as being farther than the 1-m stimulus by a factor of only $1.21(72.4 / 59.9)$. Interestingly, assuming the SDIH, a similar factor of 1.19 is implied by the size estimates $(4.4 / 3.7)$.

Both the mean and median measures suggest an asymmetry in the pattern of size responses to the small and large off-sized objects: the small off-sized objects appeared small, and the large off-sized objects tended to be seen as normal- to large-sized. The frequency data in Table 3 also show this large/small asymmetry effect. Combining the results of the 1-m and 2-m distance conditions shows that, whereas .95 of the total number of responses $(142 / 150)$ to the small off-sized objects indicated an ob-

Table 3

Frequencies of Obtained Ratios for 25 Observers in Each Distance Condition of Experiment 1

\begin{tabular}{|c|c|c|c|c|c|c|}
\hline & \multicolumn{6}{|c|}{ Distance of Object } \\
\hline & \multicolumn{3}{|c|}{$1 \mathrm{~m}$} & \multicolumn{3}{|c|}{$2 \mathrm{~m}$} \\
\hline & Normal & Small & Large & Normal & Small & Large \\
\hline \multicolumn{7}{|c|}{ Small-Sized } \\
\hline Tennis ball & 2 & 22 & 1 & 0 & 25 & 0 \\
\hline Ruler & 1 & 24 & 0 & 0 & 25 & 0 \\
\hline$\$ 5$ bill & 2 & 22 & 1 & 1 & 24 & 0 \\
\hline \multicolumn{7}{|c|}{ Normal-Sized } \\
\hline Bankcard & 10 & 11 & 4 & 5 & 19 & 1 \\
\hline Playing card & 12 & 12 & 1 & 6 & 19 & 0 \\
\hline Envelope & 8 & 14 & 3 & 4 & 21 & 0 \\
\hline \multicolumn{7}{|c|}{ Large-Sized } \\
\hline Golf ball & 11 & 5 & 9 & 10 & 12 & 3 \\
\hline Key & 10 & 2 & 13 & 12 & 8 & 5 \\
\hline Stamp & 8 & 1 & 16 & 9 & 9 & 7 \\
\hline
\end{tabular}

Note-Normal, ratio $=1$; small, ratio $<1$; and large, ratio $>1$. 
ject that was smaller than normal-sized, only .35 of the responses $(53 / 150)$ to the large objects indicated an object that was larger than normal-sized.

\section{EXPERIMENT 2}

In Experiment 1, the observers were assigned to either the 1- or 2-m distance conditions. It is possible that the perceived size ratios obtained at the 2-m distances were smaller than those at the 1 -m distances because of differences in the judgmental criteria adopted by the two groups of observers. The aim of Experiment 2 was to evaluate this possibility. A repeated measures design was used; all the observers were presented with each of the four familiar objects-two were viewed from a distance of $1 \mathrm{~m}$, and the other two from a distance of $2 \mathrm{~m}$.

\section{Method}

The observers were 16 undergraduate students, none of whom had participated in Experiment 1. The stimuli were the normal-sized bankcard, normal-sized playing card, small-sized $\$ 5$ bill, and the large-sized stamp of Experiment 1 . The bankcard and $\$ 5$ bill were viewed from a distance of $1 \mathrm{~m}$, and the playing card and stamp from a distance of $2 \mathrm{~m}$. The order of presentation of the stimuli was randomized across observers, with the restriction that each object be presented first an equal number of times. In all other respects, the procedure was identical to that of Experiment 1.

\section{Results}

The means, standard deviations, medians of the ratio scores, and the frequencies with which the 16 observers reported the objects to be small-, normal-, and large-sized are presented in Table 4 .

The pattern of results was similar to that found in Experiment 1: The normal-sized objects appeared smaller than normal at the 2-m distance and normal-sized at the $1-\mathrm{m}$ distance, the large-sized object at the 1-m distance appeared larger than normal, and the small-sized object at the 2-m distance appeared smaller than normal. Criterion differences, therefore, are unlikely to account for the difference in the pattern of results between the 1-m and 2-m distances of Experiment 1.

\section{EXPERIMENT 3}

The results of Experiment 1 indicated an asymmetry in the pattern of size responses to the large and small objects. This effect may be specific to the particular familiar objects used in the categories of large- and smallsized objects. For example, proponents of the familiarsize theory might argue that the familiar-size information of the large off-size objects was (for whatever reasons) more effective than the size information of the small offsize objects in determining perceived size. The main aim of Experiment 3, therefore, was to assess whether or not the asymmetry occurs with different-sized versions of the same familiar object. A playing card was used because the familiar-size information imparted by this object seems to be an effective determinant of direct responses to size
Table 4

Means, Standard Deviations, and Medians of Ratios of the Perceived Size of Each Familiar Object Relative to its Normal Size, and Frequencies of Obtained Ratios in Experiment 2

\begin{tabular}{|c|c|c|c|c|c|c|c|}
\hline & \multirow{2}{*}{$\begin{array}{l}\text { Viewing } \\
\text { Distance } \\
\text { (Meters) }\end{array}$} & \multicolumn{3}{|c|}{ Ratios } & \multicolumn{3}{|c|}{ Frequencies of Ratios } \\
\hline & & $M$ & $S D$ & Median & Normal & Small & Large \\
\hline \multicolumn{8}{|c|}{ Normal-Sized } \\
\hline Bankcard & 1 & .94 & .32 & .95 & 8 & 4 & 4 \\
\hline Playing card & 2 & .65 & .25 & .60 & 3 & 13 & 0 \\
\hline \multicolumn{8}{|c|}{ Off-Sized } \\
\hline Large stamp & 1 & 1.41 & .51 & 1.25 & 3 & 1 & 12 \\
\hline Small $\$ 5$ bill & 2 & .53 & .28 & .50 & 1 & 15 & 0 \\
\hline
\end{tabular}

Note-Normal, ratio $=1$; small, ratio $<1$; and large, ratio $>1$.

and to distance (e.g., Gogel, 1976; Fitzpatrick, Pasnak, \& Tyer, 1982).

The high proportion of off-sized judgments in the present Experiments 1 and 2 and in Gogel and Newton's (1969) study might be attributed to the repeated measures design of these experiments. Conceivably, the successive presentation of different-sized objects biases the responses to size by directing the observers' attention to the relative angular sizes rather than to the perceived sizes of the objects. Such a bias is likely to be even more pronounced if, as in the present experiment, the stimuli are differentsized versions of the same familiar object. These considerations dictated using a between-subjects design. For this reason, the present experiment was designed to incorporate a between-subjects component by distinguishing between the "first" and "all" trials data. The "first" trial data are the responses to the first presented card, and the "all" trials data are all the responses to a card of a particular size, irrespective of its order of presentation. By ensuring that each different-sized card was presented first an equal number of times to independent groups of observers, the "first" trial data could be analyzed by using a between-subjects design.

Unlike the preceding experiments, the observers also made judgments of the distance of the objects, which were obtained in order to assess the effectiveness of the familiarsize cue to distance. Obviously, from the perspective of the familiar-size theory, if familiar size does not influence distance judgments, then it is unlikely to influence perceived size, in which case the size results of this and the preceding experiments are of little consequence. "As noted previously, distance judgments may bias observers' reports of size. However, since the primary interest was on the effect of familiar size on the "first" trial responses to size, this problem was circumvented by obtaining the distance judgment after the observers' size response to the first presented object.

\section{Method}

The stimuli were transparencies of a normal- $(5.7 \times 8.9 \mathrm{~cm})$, large- $(1.7 \times$ normal $)$, and small-sized $(.7 \times$ normal $)$ nine-of-spades playing card. The stimuli were presented one at a time at a physical distance of either 1 or $2 \mathrm{~m}$. The angular sizes of the small, nor- 
mal, and large cards at the 1 -m distance were $2.28^{\circ}, 3.26^{\circ}$, and $5.53^{\circ}$, respectively.

There were two groups of 39 observers each. One group viewed the stimuli from a distance of $1 \mathrm{~m}$ and the other from a distance of $2 \mathrm{~m}$. The observers were undergraduate students, none of whom had participated in the previous experiments. For each distance condition, each of the three objects was presented first an equal number of times. Thus, each object was presented first to separate groups of 13 observers each. All the observers first made a single size judgment, followed by a single distance judgment of the first presented stimulus. The size instructions were identical to those of the previous experiments. After the observers made their size judgment, they were given the (unexpected) distance instruction: they were told to estimate in centimeters and meters "how far away the object appeared or seemed to be to you on your first visual impression." The observer then completed the remaining two trials. Thus, each observer made three pairs of size and distance judgmentsone pair for each of the three different-sized playing cards. In all other respects the procedure was identical to that of Experiment 1. Because of their atypical distance judgments $(8,7$, and $10 \mathrm{~m})$, the data from 3 of the observers were replaced with data from 3 additional observers.

If only the "first" trial responses are considered, the experiment reduces to a between-subjects design with six conditions, of 13 observers each, representing the factorial combination of object size (small, normal, large) and physical distance (1 or $2 \mathrm{~m}$ ). If the "all" trials responses are considered, the design is identical to the mixedsubjects design of Experiment 1, with distance as the betweensubjects factor and stimulus size as the within-subjects factor.

\section{Results}

Size. The "first" and "all" trials results are shown in Table 5. The main effects of stimulus size and distance, but not their interaction, were significant in the two-way ANOVA performed on the ratio scores of the "first" trial data $[F(2,72)=21.63 ; F(1,72)=13.76, p<.05$; $F(2,72)=1.89, p>.05$, respectively]. Identical out-

\section{Table 5}

Means, Standard Deviations, and Medians of Ratios of the Perceived Size of the Playing Card Relative to its Normal Size, and Frequencies of Obtained Ratios for the "First" and "All" Trials Data in Experiment 3

\begin{tabular}{|c|c|c|c|c|c|c|}
\hline \multirow{2}{*}{$\begin{array}{l}\text { Size of } \\
\text { Cards }\end{array}$} & \multicolumn{3}{|c|}{ Ratios } & \multicolumn{3}{|c|}{ Frequencies of Ratios } \\
\hline & $M$ & $S D$ & Median & Normal & Small & Large \\
\hline \multicolumn{7}{|c|}{ First Trials: 1-m Distance } \\
\hline Small & .57 & .32 & .50 & 2 & 11 & 0 \\
\hline Normal & .91 & .18 & 1.0 & 9 & 4 & 0 \\
\hline Large & 1.13 & .20 & 1.0 & 7 & 0 & 6 \\
\hline \multicolumn{7}{|c|}{ First Trials: $2-\mathrm{m}$ Distance } \\
\hline Small & .49 & .36 & .40 & 3 & 10 & 0 \\
\hline Normal & .54 & .27 & .50 & 2 & 11 & 0 \\
\hline Large & .90 & .21 & 1.0 & 8 & 4 & 1 \\
\hline \multicolumn{7}{|c|}{ All Trials: 1-m Distance } \\
\hline Small & .58 & .26 & .50 & 4 & 35 & 0 \\
\hline Normal & .85 & .26 & 1.00 & 17 & 16 & 6 \\
\hline Large & 1.22 & .36 & 1.30 & 13 & 4 & 22 \\
\hline \multicolumn{7}{|c|}{ All Trials: 2-m Distance } \\
\hline Small & .48 & .31 & .50 & 5 & 33 & 1 \\
\hline Normal & .65 & .29 & .50 & 4 & 32 & 3 \\
\hline Large & .89 & .28 & .95 & 16 & 16 & 7 \\
\hline
\end{tabular}

Note - Normal, ratio $=1$; small, ratio $<1$; and large, ratio $>1$. comes were obtained from the ANOVA performed on the $\log$ transformation of the magnitude estimates. The main effect of size reflects the ordering of the ratio scores across the size factor, with the highest and lowest ratio scores obtained in the large- and small-card conditions, respectively. The main effect of distance reflects the tendency for the ratio scores to be smaller for the objects at the $2-m$ than at the $1-m$ physical distance. To facilitate comparisons with the analyses of Experiment 1, the difference between the two distance conditions was assessed for each stimulus size. Relative to the $1-\mathrm{m}$ condition, the mean ratio of the $2-\mathrm{m}$ condition was significantly smaller for the large and the normal-sized cards, but not for the small card $[t(24)=2.75,4.12, p<.05 ; .57, p>.05$, respectively].

The outcomes of the two-way mixed-design ANOVA performed on the "all" trials data indicated that the main effects of distance and stimulus size, as well as their interaction, were significant $[F(1,76)=13.21 ; F(2,152)=$ $167.69 ; 9.16, p<.05$, respectively]. The interaction reflects the differential effect of increasing physical distance in reducing the magnitude of the ratio scores; the effect was greatest for the large card, smaller for the normal-sized card, and smallest for the small card. Identical outcomes were obtained in the analysis of the log transformation of the magnitude estimates. The difference between the distance conditions was significant for the normal- and large-sized-card conditions and marginally nonsignificant for the small-card condition $[t(76)=3.18$, $4.66, p<.05 ; 1.50, p=.07$, respectively].

Distance. The results are shown in Table 6. Table 6 also shows the cards' simulated distances-that is, the physical distance at which a normal-sized card subtends the same visual angle as the experimental card. There is an impressive correspondence between the distance estimates and the cards' simulated distances-the small card at the 1-m distance was reported as being farther away than the large card at the 2-m distance, and for both distance conditions the small card was reported to be farther away than the normal-sized card, which in turn was reported as being farther away than the large card. These observations are consistent with the outcomes of the ANOVAs. For both the "first" and "all" trials data, the main effect of object size was significant $[F(2,72)=$ $14.46 ; F(2,152)=92.94, p<.05$, respectively], as was the main effect of physical distance $[F(1,72)=25.05$; $F(1,72)=26.25, p<.05]$. The interaction between object size and distance was significant in the analysis of the "all" but not the "first" trials data $[F(2,152)=6.75$, $p<.05 ; F(2,72)=1.21, p>.05$, respectively].

The main effect of physical distance cannot be attributed to the effectiveness of residual distance cues. The results of the control condition of Experiment 1 suggest that the objects at the $2-\mathrm{m}$ distance were perceived as being farther away than the objects at the 1-m distance by a factor of about 1.2. In fact, the cards at the $2-\mathrm{m}$ distance were reported as being farther away than the cards at the $1-\mathrm{m}$ distance by a factor of almost 2.0 , as might be expected 
Table 6

Means, Standard Deviations, and Medians (in Meters) of Distance Estimates of the Playing Cards for the "First" and "All" Trials Data, and the Cards' Simulated Distances (in Meters) in Experiment 3

\begin{tabular}{|c|c|c|c|c|c|c|c|c|}
\hline \multirow[b]{3}{*}{$\begin{array}{l}\text { Size of } \\
\text { Cards }\end{array}$} & \multicolumn{8}{|c|}{ Viewing Distance } \\
\hline & \multicolumn{4}{|c|}{$1 \mathrm{~m}$} & \multicolumn{4}{|c|}{$2 \mathrm{~m}$} \\
\hline & $M$ & $S D$ & Median & $\begin{array}{c}\text { Simulated } \\
\text { Distance }\end{array}$ & $M$ & $S D$ & Median & $\begin{array}{c}\text { Simulated } \\
\text { Distance } \\
\end{array}$ \\
\hline \multicolumn{9}{|c|}{ First Trials } \\
\hline Small & 1.20 & .43 & 1.00 & 1.43 & 2.22 & 1.22 & 2.00 & 2.86 \\
\hline Normal & .78 & .38 & .70 & 1.00 & 1.47 & .62 & 1.30 & 2.00 \\
\hline Large & .53 & .17 & .50 & .59 & .99 & .44 & 1.00 & 1.18 \\
\hline \multicolumn{9}{|c|}{ All Trials } \\
\hline Small & 1.18 & .54 & 1.00 & 1.43 & 2.12 & 1.18 & 2.00 & 2.86 \\
\hline Normal & .81 & .43 & .75 & 1.00 & 1.47 & .71 & 1.50 & 2.00 \\
\hline Large & .52 & .33 & .50 & .59 & .96 & .52 & 1.00 & 1.18 \\
\hline
\end{tabular}

if familiar size is an effective distance cue. For example, the "first" trial distance estimates indicate that the small, normal-sized, and large cards at the 2-m distance were judged to be $1.85,1.88$, and 1.86 times the distance of the corresponding objects at the $1-\mathrm{m}$ distance.

\section{Discussion}

The distance results clearly show that familiar size is an effective determinant of responses to distance. Although this finding is hardly novel (e.g. , Fitzpatrick et al., 1982; Gogel \& Da Silva, 1987; Predebon, 1992b), its significance for the familiar-size theory is that it makes it difficult to defend the theory by dismissing the size findings on the grounds that familiar size was an ineffective determinant of reports of distance and, by implication via the SDIH, perceived size.

Of principal interest are the size results. As is evident from the ratio data in Table 5, the "first" trial results are similar to the "all" trials results. The small card appeared to be small-sized at the 1-m distance and there was a tendency for it to be reported as being even smaller at the 2-m distance; the normal-sized card appeared normalto small-sized at the $1-\mathrm{m}$ distance and small-sized at the 2-m distance; and the large card appeared normal- to large-sized at the 1-m distance and normal-to small-sized at the 2-m distance. The frequency data, however, do suggest some difference between the "first" and "all" trials conditions. Specifically, the proportion of responses falling in the normal-sized category is higher for the "first" (.40) than for the "all" (.25) trials responses, although allowance must be made for the relatively small sample size of the "first" trials condition. Importantly, the general similarity between the "first" and "all" trial results seems to exclude an explanation of the "all" trials results of the present experiment and of the results of Experiments 1 and 2 solely in terms of the observers' responding to the relative angular sizes of the successively presented objects.

The mean and median measures confirm the small/large asymmetry effect found in Experiment 1: The small card at both distances was perceived to be smaller than normal- sized, whereas the large card tended to be perceived as being slightly larger than normal at the $1-\mathrm{m}$ distance and normal- to small-sized at the 2-m distance. The asymmetry is also evident in the frequency data shown in Table 5. Combining the data of the 1- and 2-m conditions, the proportion of "small" off-sized responses to the small card and the proportion of "large" off-sized responses to the large card are .81 (21/26) and .27 (7/26), respectively, for the "first"' trial responses $(26=2$ distances $\times 13$ subjects), and the corresponding proportions for the "all" trials responses ( $78=2$ distances $\times 39$ subjects) are .87 $(68 / 78)$ and $.37(29 / 78)$, respectively.

\section{SUPPLEMENTARY EXPERIMENT}

The asymmetry effect might be attributed, at least partly, to nonperceptual factors. Perhaps observers are less likely to report perceiving an object that is larger than normal-sized, since such perceptions are rarely experienced under naturalistic viewing conditions (Predebon, 1990). In contrast, perceptions of familiar objects as being smaller than normal-sized are experienced frequently (e.g., a person viewed at an appreciable distance is said to appear doll-like), and this is reflected by the widespread belief-the perspective bias (Carlson, 1977) that there is an inverse relationship between the perceived size of an object and its distance. Although the instructions in the present experiments requested judgments of apparent size, they did not specifically exclude judgments based on the observers' assessment of the actual or objective size of the object. Given that observers sometimes find it difficult to distinguish between the concepts of objective and apparent size (e.g., Predebon, Wenderoth, \& Curthoys, 1974), and given that large off-sized perceptions of familiar objects occur infrequently in naturalistic viewing environments, some observers may have assumed, and reported, the large-sized objects to be normal-sized, attributing the large off-sized perception to the inferred distance of the object. Ten observers who had not participated in the previous experiments were run in the $2-\mathrm{m}$ distance condition of Experiment 3. The instructions were similar to those of the main experiments, except that they included the following statements: "I am not interested in what you think is the actual size of the card, that is, whether you think the card is physically normal-sized or physically off-sized. . . . Just make your judgment on how the object looks or seems to be to you on your first visual impression." Additionally, the distinction between the actual and the apparent size of the objects was reinforced by giving examples, such as the apparent and actual size of the moon.

The results were similar to the 2-m distance condition of Experiment 3. The frequency of reports of a small-, normal-, and large-sized card were 1,9 , and 0 for the small-sized card, and the corresponding frequencies for the normal- and large-sized cards were 2, 8, and 0 , and 5, 3 , and 2 , respectively. The means (and medians in parentheses) of the ratio scores were $.47(.40), .63(.60)$, and 
$.96(1.00)$ for the small-, normal-, and large-sized cards, respectively. It seems reasonable to conclude from these findings that the asymmetry effect cannot be attributed to the failure of the observers in the main experiments to understand the notion of the apparent size.

\section{SUPPLEMENTARY DATA ANALYSIS}

According to the theory of off-sized perceptions, there should be a positive association between the perceived sizes of the objects and the sizes of their visual angles. The size instructions did not request judgments of perceived size per se, however; rather, they requested a judgment of the ratio of the perceived size of the object relative to its remembered normal size. Nevertheless, an estimate of perceived size can be obtained by multiplying the mean size judgment (ratio score) of each object by the object's normal size. Figure 1 presents scatter diagrams to illustrate the relationship between the estimated perceived sizes and the sizes of the objects' visual angles. Figure 1A shows the data of all 13 conditions of Experiments 1 and 2. The Pearson product-moment correlation is significant $(r=.87, p<.05)$, indicating a positive association between visual angle size and estimated size. An association between estimated size and visual angle is to be expected, however, since there is a correlation $(r=.56, p<.05)$ between visual angle size and the normal sizes of the familiar objects. ${ }^{3}$ This confound does not occur in Experiment 3, in which different-sized versions of the same familiar object were used. As indicated in Figure 1B, which shows the "all" trials data of Experiment 3 , there is an association $(r=.96, p<.05)$ between estimated size and visual angle size, and a similar association exists for the the "first" trial results $(r=.93$, $p<.05)$.

\section{GENERAL DISCUSSION}

The results confirm Gogel's (1969) and Gogel and Newton's (1969) finding that, under visually impoverished conditions, familiar objects are frequently perceived to be off-sized. Additionally, there are two important new findings-the frequency of off-sized judgments is influenced by the object's visual angle, and there is an asymmetry between the size responses to the large and small objects. In general, the objects were reported to be smaller at the $2-\mathrm{m}$ distance than they were at the $1-\mathrm{m}$ distance, and reports of off-sized perceptions occurred more frequently for the small objects than for the large objects.

The large/small asymmetry effect found in Experiment 1 is not specific to the familiar objects that were used in the large- and small-sized categories, since a similar asymmetry was found in Experiment 3, in which different-sized versions of the same familiar object were used. Nor is the effect specific to the conditions of the present investigation. Inspection of Gogel and Newton's (1969) data indicates a similar asymmetry. Of the 288 (32 subjects $\times$
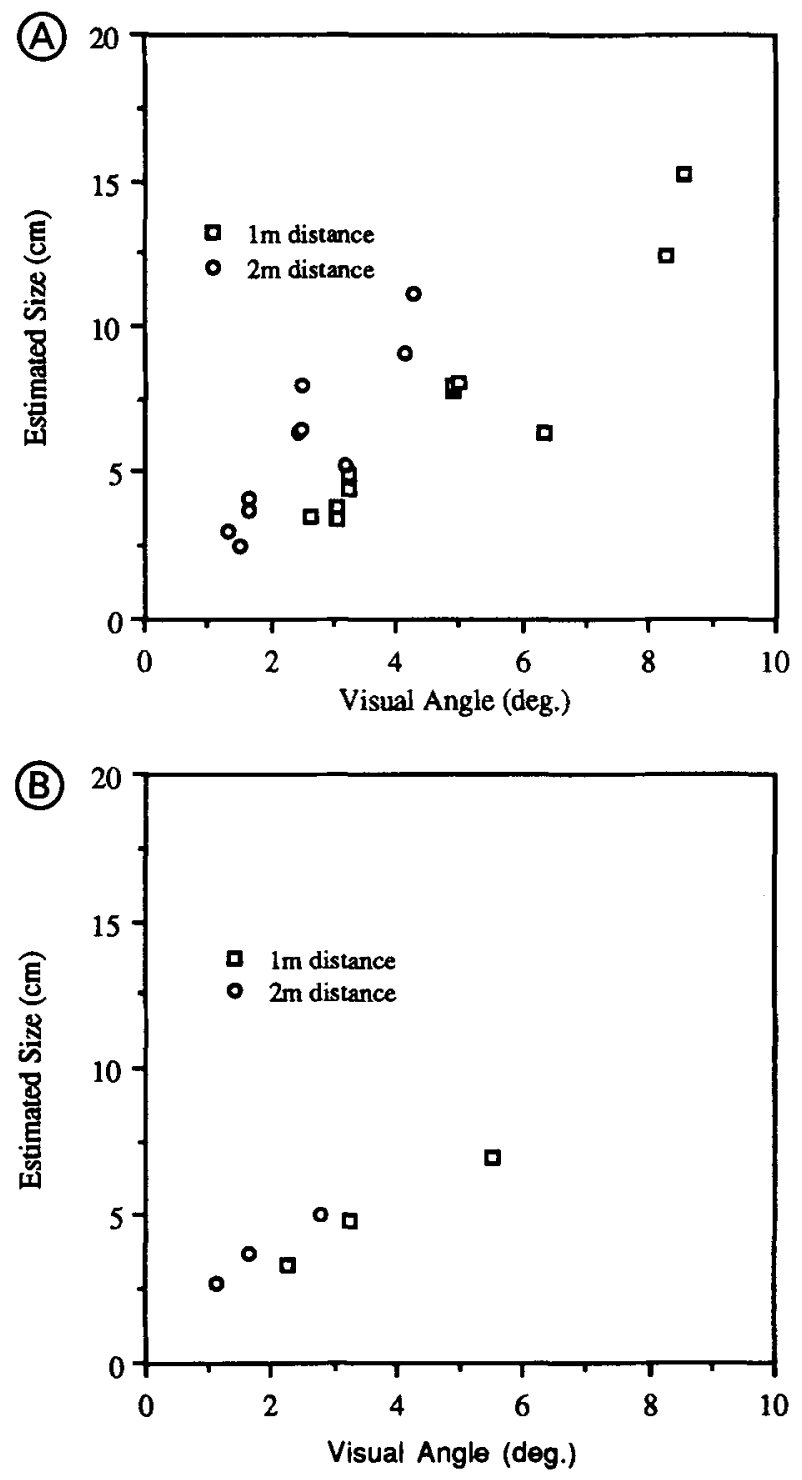

Figure 1. Scatter diagrams illustrating the relationship beween the estimated size and the visual angle size of the objects in Experiments 1 and 2 (A) and Experiment 3 (B).

9 objects) responses to the size of the small objects, .14 $(39 / 288)$ and $.84(241 / 288)$ were reports of a normal- and small-sized object, respectively, and of the 224 (32 subjects $\times 7$ objects) responses to the size of the large objects, $.49(109 / 224)$ and $.27(62 / 224)$ were reports of a normal- and large-sized object, respectively. It seems, then, that for the range of physical distances examined in the present investigation (1 and $2 \mathrm{~m}$ ) as well as in Gogel and Newton's study $(1.3 \mathrm{~m})$, there was a tendency for the subjects to perceive large off-sized objects more frequently as normal- rather than large-sized, and to perceive small off-sized objects more frequently as small- rather than normal-sized.

The results of Experiment 3 indicate that familiar size strongly influences judgments of distance. If these judg- 
ments are construed as reflecting observers' distance perceptions, then in agreement with the SDIH, both the normal- and off-sized objects should be frequently reported as appearing normal-sized. Yet, a high proportion of the judgments indicated otherwise. For example, in Experiment $3, .60$ of the 78 "first" trial responses ( 3 objects $\times 2$ distances $\times 13$ subjects) and .75 of the 234 "all" trial responses ( 3 objects $\times 2$ distances $\times 39$ subjects) were of an off-sized card. The strong influence of familiar size on reports of distance on the one hand, and its weak effect on reports of perceived size on the other, is inconsistent with the strong form of the familiar-size theory. Rather, if it is assumed that reports of distance include a cognitive component of the kind posited by the theory of off-sized perceptions, then the pattern of size and distance reports might be interpreted as supporting the weak form of the familiar-size theory.

However, aspects of the size results are inconsistent with the weak version of the familiar-size theory. This version predicts a correspondence between reports of offsized perceptions and the category size of the objects: Although the perceived size of the objects are assumed to regress toward the object's normal size, the physically normal-, large-, and small-sized objects should still appear, and be reported as normal-, large-, and small-sized, respectively. In fact, the normal-sized objects frequently were reported as being small-sized, and the large-sized objects as being either normal- or small-sized. Moreover, the weak form of the theory seems to be inconsistent with the subjects' tendency to perceive the objects, including the physically normal-sized objects, as being smaller at the 2-m than at the $1-\mathrm{m}$ physical distance.

As noted previously, the effect of familiar size on reports of distance is consistent with both the theory of offsized perceptions and the familiar-size theory, although the former, unlike the latter, interprets the effect as a consequence of an off-sized perception's eliciting a cognitive response to distance. More importantly, the size results support the theory of off-sized perception. The asymmetry effect, the association between visual angle and estimated size, and, in particular, the effect of physical distance on the pattern of off-sized responses to the objects in the three different size categories, are readily explicable by the theory. Specifically, if it is assumed that the objects are localized at an ERD of less than about $1 \mathrm{~m}$, it follows from the SDIH that the physically normal-sized objects will appear normal-sized or slightly smaller than normal-sized at the 1-m distance and small-sized at the 2-m distance, the physically small-sized objects will appear smaller than normal at both distances with a greater underestimation of perceived size at the 2-m distance, and the large objects will appear normal-to large-sized at the $1-\mathrm{m}$ distance and normal- to small-sized at the $2-\mathrm{m}$ distance.

Independent evidence that the ERD localized the objects at a relatively near distance is provided by the results of the control condition of Experiment 1. However, if the objects were perceived at a constant ERD irrespec- tive of their physical distance of presentation, then the objects at the 2-m distance should be perceived as being twice as large as the same objects at the $1-\mathrm{m}$ distance. As is evident from the ratio data in Tables 2 and 5 , this generally did not occur. This discrepancy might be explained by assuming that the ERD was somewhat less than $1 \mathrm{~m}$ and greater than $1 \mathrm{~m}$ for the $1-\mathrm{m}$ and $2-\mathrm{m}$ physical distance conditions, respectively, perhaps because of the influence of residual oculomotor distance cues. Although viewing was monocular, it seems reasonable to suppose that accommodation and convergence did vary somewhat as a function of the physical distance of the objects, in which case the ERD would be slightly greater for the objects at the 2-m distances than at the 1-m distances. Consistent with this possibility, the results of the control condition suggested a small effect of residual distance cues with the 2 -m objects perceived to be about 1.2 times the distance of the 1-m objects. ${ }^{4}$

The size results of the present investigation support the conclusions of a number of studies that have used different procedures to evaluate the effectiveness of the familiarsize cue to perceived size. For example, the exponent of the power function relating perceived size to physical size is unaffected by whether or not the objects of the series have a familiar size (Predebon, 1992b), and experimental instructions that request judgments of perceived size (apparent instructions) typically yield either a very weak or a nonexistent effect of familiar size on direct reports of size (Gogel \& Da Silva, 1987; Higashiyama, 1984; Predebon, 1992a).

A potential limitation of the present findings needs to be considered. The observers may have recognized the stimuli as photographic depictions of familiar objects, and this might explain the high frequency of off-sized judgments. Arguably, if familiar size affects perceived size and distance differently for photographic depictions and real objects, the present results have little bearing on the role of familiar size in determining the spatial characteristics of objects. This possibility is discounted for a number of reasons. For one, the realistic color reproductions were viewed under completely darkened conditions, and they looked like real objects suspended against an undifferentiated black background. For another, a few of the observers in the present experiments, as well as observers from other unpublished experiments that used some of the stimuli of the present investigation, spontaneously inquired as to how the experimenter illuminated the objects, implying that the stimuli were virtually indistinguishable from real objects.

In summary, neither the weak nor the strong versions of the familiar-size theory is able to explain parsimoniously the pattern of size results and, in particular, the effect of visual angle and the category size of objects on reports of off-sized perceptions. In contrast, the findings are consistent with (and readily predictable from) the theory of off-sized perceptions and, more generally, with the theory's claim that familiar size is not an important datum of perceptual experience. There is one major "meta- 
theoretical" qualification to this conclusion. Both the familiar-size theory and the theory of off-sized perceptions assume the validity of the SDIH. However, if perceived size and perceived distance are not linked in the manner specified by the SDIH, then quite a different account of the present findings is mandated. Specifically, while the findings permit the conclusion that familiar size is unlikely to be a major determinant of perceived size, they do not exclude a role of familiar size in determining perceived distance. Viewed in this context, the distance results of Experiment 3 may very well reflect an effect of familiar size on perceived distance. The issue of whether or not familiar size influences perceived distance, as distinct from eliciting a cognitive response to distance, is beyond the scope of the present work; obviously, independent converging evidence is required to resolve the reports of distance into perceptual and cognitive components.

\section{REFERENCES}

Carlson, V. R. (1977). Instructions and perceptual constancy judgments. In W. Epstein (Ed.), Stability and constancy in visual perception: Mechanisms and processes (pp. 217-254). New York: Wiley.

FitzPatrick, V., Pasnak, R., \& TYer, Z. E. (1982). The effect of familiar size at familiar distances. Perception, 11, 85-91.

GoGEL, W. C. (1969). The effect of object familiarity on the perception of size and distance. Quarterly Journal of Experimental Psychology, 21, 239-247.

GoGEL, W. C. (1976). An indirect method of measuring perceived distance from familiar size. Perception \& Psychophysics, 20, 419-429.

Gogel, W. C., \& DA Silva, J. A. (1987). Familiar size and the theory of off-sized perceptions. Perception \& Psychophysics, 41, 318-328.

GoGel, W. C., \& Newton, R. E. (1969). Perception of off-sized objects. Perception \& Psychophysics, 5, 7-9.

HigashiYama, A. (1984). The effects of familiar size on judgments of size and distance: An interaction of viewing attitude with spatial cues. Perception \& Psychophysics, 35, 305-312.

ITTELSON, W. H. (1960). Visual space perception. New York: Springer.

Predebon, J. (1990). Relative distance judgments of familiar and unfamiliar objects viewed under representatively natural conditions. Perception \& Psychophysics, 47, 342-348.

Predebon, J. (1992a). The influence of object familiarity on magnitude estimates of apparent size. Perception, 21, 77-90.

Predebon, J. (1992b). The role of instructions and familiar size in absolute judgments of size and distance. Perception \& Psychophysics, 51, 344-354.
Predebon, J., Wenderoth, P. M., \& Curthoys, I. S. (1974). The effects of instructions and distance on judgments of off-size familiar objects under natural viewing conditions. American Journal of Psychology, 87, 425-439.

SEDGWICK, H. A. (1986). Space perception. In K. R. Boff, L. Kaufman, \& J. P. Thomas (Eds.), Handbook of perception and human performance: Vol 1. Sensory processes and perception (pp. 21-1-2157). New York: Wiley.

\section{NOTES}

1. Although Gogel and Newton (1969) argued that the specific-distance tendency, and not residual distance cues, determined the perceived distance and therefore the perceived size of the unfamiliar object, they did not provide independent evidence for their claim.

2. For example, if the ERD is $1.5 \mathrm{~m}$, the normal-sized objects at the 1- and 2-m distances should appear larger and smaller, respectively, than normal-sized. Similarly, if the ERD is $4 \mathrm{~m}$, the normal-sized objects at $1 \mathrm{~m}$ should appear twice as large as the objects at the 2-m distance.

3. The correlation between the objects' normal size and visual angle size is even higher when the $1-\mathrm{m}$ and $2-\mathrm{m}$ distance conditions are considered separately $(r=.79, .77, p<.05$, respectively).

4. If the objects at the 2-m distance are localized at a distance of about $1 \mathrm{~m}$, then both the small and normal-sized objects should appear smaller than normal, and the large objects should appear close to their normal size. In effect, then, for the small and normal-sized objects at the 2-m distance, the observers were constrained to using numerical values less than about 100 (i.e., the value assigned to the remembered normal size of the object). The somewhat higher than expected size ratios for the 2 -m distance conditions might be explained in terms of a "floor" effect: The observers tended to avoid using numbers (e.g., 5, 10) near the bottom end of the response continuum. An independent group of 10 observers were first shown a piece of white cardboard-the standard (S) - of the same size $(5.7 \mathrm{~cm}$ wide) and shape as a playing card, and told to assign a value of 100 to its apparent size. The standard was then removed, and the observers made judgments of the apparent size of two stimuli $(.30 \times \mathrm{S}$ and $.65 \times \mathrm{S})$ of the same shape as the standard. The stimuli were attached to a light-gray cardboard background, and were viewed binocularly under full-cue conditions from a distance of $2 \mathrm{~m}$. As in the present investigation, the observers were instructed to assign a number to the apparent size of the stimulus relative to the size (100) of the standard. The means and standard deviations (in parentheses) of the ratio scores (judged size/100) were $.42(.18)$ and $.73(.17)$ for the $.3 \times \mathrm{S}$ and $.65 \times \mathrm{S}$ stimuli, respectively. These results suggest that the higher-than-expected values of the ratio scores of the familiar objects at the 2-m distance conditions may, at least partly, reflect either a tendency for observers to avoid numbers at the lower end of the range of allowable numbers, or a tendency to overestimate the size of objects that are smaller than the remembered size of the standard stimulus.

(Manuscript received April 12, 1993; revision accepted for publication January $18,1994$. ) 